\title{
Effects of resveratrol alone or in combination with piperine on cerebral blood flow parameters and cognitive performance in human subjects: a randomised, double-blind, placebo-controlled, cross-over investigation
}

\author{
Emma L. Wightman ${ }^{1 *}$, Jonathon L. Reay ${ }^{2}$, Crystal F. Haskell ${ }^{1}$, Gary Williamson ${ }^{3}$, Tristan P. Dew ${ }^{3}$ \\ and David O. Kennedy ${ }^{1}$ \\ ${ }^{1}$ Brain, Performance and Nutrition Research Centre, Northumbria University, Newcastle upon Tyne NE1 8ST, UK \\ ${ }^{2}$ School of Social Sciences and Law, Teesside University, Middlesbrough TS1 3BA, UK \\ ${ }^{3}$ School of Food Science and Nutrition, University of Leeds, Leeds LS2 9JT, UK
}

(Submitted 26 April 2013 - Final revision received 23 December 2013 - Accepted 23 January 2014 - First published online 7 May 2014)

\section{Abstract}

Previous research has shown that resveratrol can increase cerebral blood flow (CBF) in the absence of improved cognitive performance in healthy, young human subjects during the performance of cognitively demanding tasks. This lack of cognitive effects may be due to low bioavailability and, in turn, reduced bioefficacy of resveratrol in vivo. Piperine can alter polyphenol pharmacokinetics, but previous studies have not investigated whether this affects the efficacy of the target compound. Therefore, the objective of the present study was to ascertain whether co-supplementation of piperine with resveratrol affects the bioavailability and efficacy of resveratrol with regard to cognition and CBF. The present study utilised a randomised, double-blind, placebo-controlled, within-subjects design, where twenty-three adults were given placebo, trans-resveratrol $(250 \mathrm{mg}$ ) and trans-resveratrol with $20 \mathrm{mg}$ piperine on separate days at least a week apart. After a $40 \mathrm{~min}$ rest/absorption period, the participants performed a selection of cognitive tasks and CBF was assessed throughout the period, in the frontal cortex, using near-IR spectroscopy. The presence of resveratrol and its conjugates in the plasma was confirmed by liquid chromatography-MS analysis carried out following the administration of the same doses in a separate cohort $(n 6)$. The results indicated that when co-supplemented, piperine and resveratrol significantly augmented CBF during task performance in comparison with placebo and resveratrol alone. Cognitive function, mood and blood pressure were not affected. The plasma concentrations of resveratrol and its metabolites were not significantly different between the treatments, which indicates that co-supplementation of piperine with resveratrol enhances the bioefficacy of resveratrol with regard to CBF effects, but not cognitive performance, and does this without altering bioavailability.

\section{Key words: Resveratrol: Piperine: Near-IR spectroscopy: Cognitive performance: Cerebral blood flow}

Resveratrol (3,5,4'-trihydroxystilbene) is a polyphenolic secondary metabolite produced within plants in response to a range of environmental stressors ${ }^{(1)}$. Resveratrol ingestion has also been shown to have protective effects in animals and human subjects. Of direct relevance here is that these effects include the protection of cognitive function/reversal of cognitive deficits in animal models following supplementation ${ }^{(2)}$, which may, in large part, be due to the cerebral blood flow (CBF) effects exerted by resveratrol ${ }^{(3)}$. These CBF effects are likely to be mediated by the ability of resveratrol to modulate NO synthesis ${ }^{(4)}$, with oral intervention shown to enhance endothelium-dependent relaxation in rats ${ }^{(5,6)}$ and improve flow-mediated dilatation in overweight/obese human subjects $^{(7)}$. An increase in blood-borne neural metabolic substrates such as oxygen ${ }^{(8)}$ and glucose ${ }^{(9)}$ has been reported to enhance aspects of cognitive performance in healthy, young human subjects. Taken together, it could be hypothesised that an acute increase in CBF, augmenting the delivery of metabolic substrates, might also beneficially affect cognitive performance.

A recent study carried out in our laboratory has demonstrated a dose-related increase in prefrontal cortex CBF during the performance of cognitively demanding tasks in healthy, young adults. This effect was consistent across all time points for $500 \mathrm{mg}$ of resveratrol, but failed to reach significance for $250 \mathrm{mg}$. The increase in CBF did not facilitate improved cognitive task performance ${ }^{(10)}$. It was argued that this might be due to the low bioavailability of resveratrol.

The pepper-derived alkaloid piperine has been observed to be a potent enhancer of the bioavailability of numerous compounds, including polyphenols, in vivo, for instance, epigallocatechin-3-gallate in rodents ${ }^{(11)}$, curcumin in rats

Abbreviations: CBF, cerebral blood flow; NIRS, near-IR spectroscopy; RVIP, rapid visual information processing.

*Corresponding author: E. L. Wightman, email emma.l.wightman@northumbria.ac.uk 
and human subjects ${ }^{(12)}$, and $\beta$-carotene following $14 \mathrm{~d}$ of co-supplementation in human subjects ${ }^{(13)}$. Co-supplementation of piperine with resveratrol $(10 \mathrm{mg} / \mathrm{kg})$ has been reported to induce $1544 \%$ enhancement of maximum serum resveratrol levels (compared with $100 \mathrm{mg} / \mathrm{kg}$ resveratrol alone) and increase exposure (AUC) by $229 \%$ in mice ${ }^{(14)}$. Potential mechanisms for these phenomena include inhibition of enzymes responsible for the metabolism of polyphenols ${ }^{(14-16)}$, enhancement of metabolism via thermogenic effects ${ }^{(13)}$ and/ or competition for membrane efflux pumps in the body and brain: phenomena observed when plant-derived compounds are co-administered, e.g. polyphenols ${ }^{(17)}$. However, these studies did not investigate whether increased bioavailability leads to increased bioefficacy of the target compound.

Therefore, the present randomised, double-blind, placebocontrolled, cross-over study investigated the effects of $250 \mathrm{mg}$ resveratrol when administered alone and when co-supplemented with $20 \mathrm{mg}$ piperine. The rationale for using $250 \mathrm{mg}$ resveratrol in the present study is based on the previous ineffectiveness of this dose in modulating CBF and the expectation that this will be augmented by the actions of piperine. The aim was to ascertain whether piperine is capable of enhancing the bioefficacy of resveratrol with regard to $\mathrm{CBF}$ and cognitive performance in healthy adults. Blood plasma concentrations of resveratrol were measured to investigate whether bioavailability correlated with bioefficacy.

\section{Experimental methods}

\section{Participants (cerebral blood flow and cognitive performance assessment)}

A total of twenty-three healthy adults (four males and nineteen females, mean age 21 years, range $19-34$ years, SD $3 \cdot 2$ years, all right handed) took part in all the three arms of the crossover study. The data collected from one participant were excluded from the analysis due to data catchment errors. All participants visited the laboratory after a $12 \mathrm{~h}$ overnight fast and reported to meet the inclusion criteria, i.e. to be in good health and free from social drug, alcohol, prescription medication, and herbal extract/food supplement use, relevant food allergies, intolerances and digestive problems. A fasted state was considered to be most appropriate due to the individual differences involved in breakfast consumption and the unknowns involved in the absorption of resveratrol together with food. Although food deprivation has been reported to deleteriously affect cognitive function previously in children ${ }^{(18,19)}$, more recent research in athletes during Ramadan has been more ambiguous ${ }^{(20)}$ and a well-controlled study of healthy, young adults has found no detrimental effects of fasting on cognitive performance ${ }^{(21)}$. All participants were non-smokers and did not consume excessive amounts of caffeine ( $>6$ cups of coffee or equivalent/d). In addition, participants who had suffered a head injury, neurological disorder or neurodevelopmental disorder were excluded from participation, as were those who had uncorrected sight problems or were pregnant or seeking to become so.

\section{Participants (bioavailability assessment)}

In the bioavailability analysis, six healthy (mean BMI $24.2 \mathrm{~kg} / \mathrm{m}^{2}$, range $21 \cdot 7-27 \cdot 2 \mathrm{~kg} / \mathrm{m}^{2}$, SD $2 \cdot 38 \mathrm{~kg} / \mathrm{m}^{2}$ ) male adults (mean age $25 \cdot 8$ years, range $23-29$ years) took part. Inclusion/ exclusion criteria were as per the $\mathrm{CBF}$ and cognitive performance aspect of the study.

The present study was conducted according to the guidelines laid down in the Declaration of Helsinki, and all procedures involving human subjects were approved by the Department of Psychology ethics committee of Northumbria University. Written informed consent was obtained from all subjects. The present trial was registered at ClinicalTrials.gov (study identifier NCT01331382).

\section{Treatments}

During the three study visits, the participants received three single-dose treatments in an order dictated by random allocation to a counterbalancing (Latin square) order. The three treatments comprised two capsules, with each combination delivering an inert placebo, $250 \mathrm{mg}$ of trans-resveratrol or $250 \mathrm{mg}$ of trans-resveratrol plus $20 \mathrm{mg}$ of piperine. The treatments were administered in identical size 0 vegetable capsules, which were prepared by the lead researcher and coded by a third party who had no further involvement in any aspect of the study. No member of the investigational team was aware of the contents of the capsules until a blind-data review was completed.

\section{Near-IR spectroscopy}

Relative changes in the absorption of near-infrared light were measured at a time resolution of $10 \mathrm{~Hz}$ using a twelve-channel Oxymon system (Artinis Medical Systems B.V.). The system emitted two nominal wavelengths of light (approximately 765 and $855 \mathrm{~nm}$ ) with an emitter/optode separation distance of $4 \mathrm{~cm}$. The differential pathlength factor was adjusted according to the age of the participant. Relative changes in the concentrations of oxy- $\mathrm{Hb}$, deoxy- $\mathrm{Hb}$ and total $\mathrm{Hb}$ were calculated by means of a modified Beer-Lambert law ${ }^{(22)}$ using the proprietorial software. Given the extended recording period and the investigational aims, a simple two-emitter/optode pair configuration was used (i.e. two channels). The emitter/optode pairs were positioned over the left and right frontal cortices using a standard optode holder headband, which separated the pairs from each other by $4 \mathrm{~cm}$. Therefore, each pair collected data from an area of prefrontal cortex that included the areas corresponding to the International 10-20 system Fp1 and Fp2 electroencephalogram positions. The near-IR spectroscopy (NIRS) data output was time stamped at the start of each task segment to ensure that the data corresponded to the relevant epoch of task performance.

\section{Cognitive tasks}

To maximise the cerebral activity-induced modulation of blood flow, a pilot study was initially carried out with a 
separate cohort of fifteen participants (three males and twelve females, mean age 21.6 years, all right handed) to ascertain the most 'mentally demanding' and 'difficult' tasks from a battery of eleven tasks (data not reported). The five tasks used in the study were all subjectively rated as both the most 'demanding' and most 'difficult' and have all previously been shown to activate the frontal cortex in functional MRI studies $^{(23-25)}$. A computerised battery of cognitive tasks were delivered using the Computerised Mental Performance Assessment System software.

Serial subtractions. The serial subtraction task consisted of 2 min each of serial $7 \mathrm{~s}, 13 \mathrm{~s}$ and $17 \mathrm{~s}$. The task has been described in detail by Kennedy et al. ${ }^{(10)}$.

Rapid visual information processing. The rapid visual information processing (RVIP) task has been described in detail by Kennedy et al. ${ }^{(10)}$.

$\mathrm{N}$-back task. The three-back version of the $\mathrm{N}$-back task was used in this paradigm, requiring the participants to indicate whether the letter presented on screen was also present three-letter back in the letter sequence. The participants were required to respond by pressing the 'yes' or 'no' button on the response box, to each letter, as quickly as they could. This task includes sufficient stimuli (letters) to last for at least $2 \mathrm{~min}$, although this is dependent on speed (i.e. slower reaction times will result in a lengthier task) and is scored for accuracy and reaction time.

Mood visual analogue scales. The participants were required to rate how 'relaxed', 'alert', 'jittery', 'tired', 'tense' and 'mentally fatigued' they felt by placing a cross with the mouse and cursor on a $100 \mathrm{~mm}$ on-screen line between the descriptors 'not at all' and 'extremely'. They were also required to rate their 'overall mood' on a scale anchored by 'very poor' to 'very good' and their levels of 'headache' between 'not at all' and 'extremely'. The visual analogue scales were scored as a percentage along the line denoting more of the relevant adjective.

\section{Procedure (cerebral blood flow and cognitive performance assessment)}

Each participant was required to visit the laboratory on four occasions. The first of these was an initial screening/training visit during which the participants provided written informed consent, were screened with regard to the study inclusion/ exclusion criteria, briefed with regard to compliance requirements and given training in completing the cognitive tasks. This visit was followed within $14 \mathrm{~d}$ by the first of three active study morning sessions.

On each of the three active study morning sessions, which were conducted $2-14 \mathrm{~d}$ apart, the participants visited the laboratory at 08.30 hours in a fasted state and provided confirmation of continued compliance with regard to the inclusion/exclusion criteria. After a $5 \mathrm{~min}$ seated resting period, a blood pressure reading was taken, after which the NIRS headband was fitted. The participants then completed a series of mood visual analogue scales and two repetitions of baseline cognitive tasks in the following order: serial $7 \mathrm{~s}$; RVIP; serial $13 \mathrm{~s} ; \mathrm{N}$-back; serial $17 \mathrm{~s}$. The participants then rested for $10 \mathrm{~min}$ and a second blood pressure reading was taken. Treatment was then administered, after which the participants sat quietly, watching one of a selection of non-arousing DVD for a 40 min 'absorption' period. Following this, a third blood pressure reading was taken, after which the participants completed four repetitions of the aforementioned tasks in the same order and duration. After the completion of the post-dose tasks, the same mood visual analogue scales were presented and the fourth and final blood pressure readings were taken. NIRS data were captured throughout the

NIRS recording
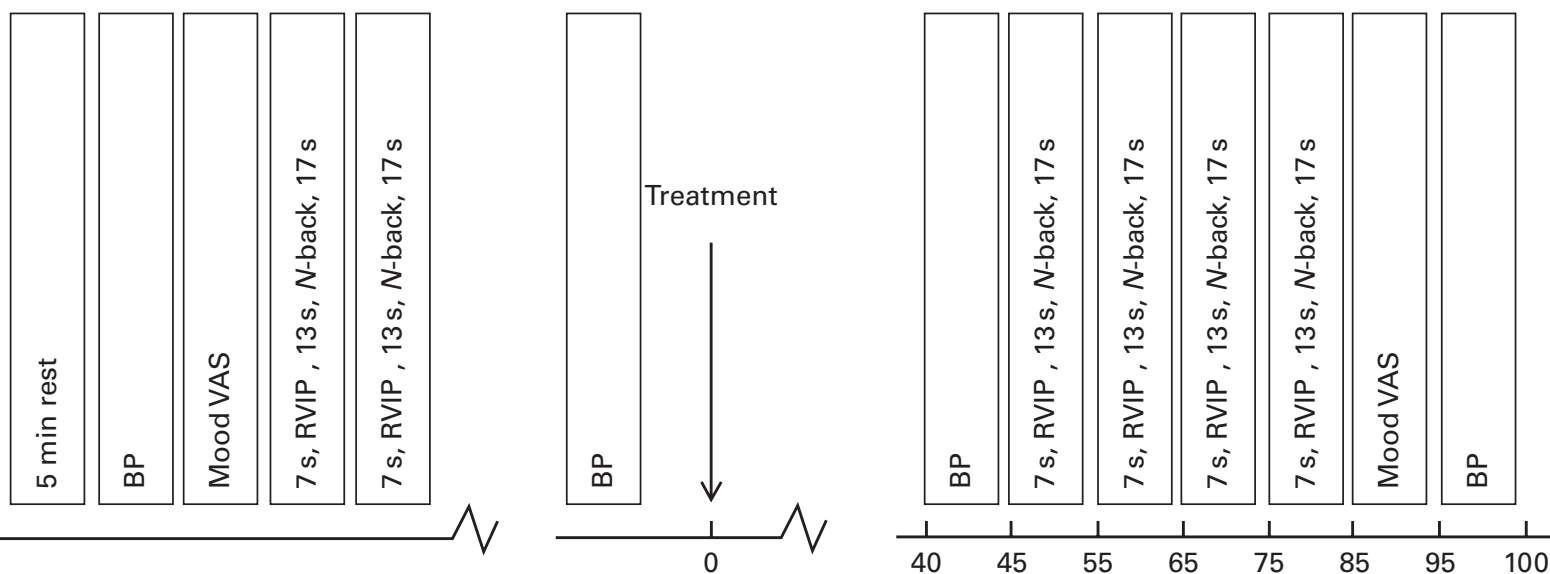

Fig. 1. Timeline and running order of the test sessions. Upon arrival to the laboratory, the participants rested for 5 min before the first blood pressure (BP) reading was taken. The near-IR spectroscopy (NIRS) headband was then fitted. Mood visual analogue scales (VAS) and two repetitions of baseline cognitive tasks were completed, followed by a $10 \mathrm{~min}$ rest period. The second blood pressure reading was then taken and treatment was administered. After a 40 min absorption period, the third blood pressure reading was taken. Later, four repetitions of the cognitively demanding tasks were completed, followed by mood VAS ratings and the fourth and final blood pressure readings. RVIP, rapid visual information processing. 
sessions. The timeline and running order of the test sessions are shown in Fig. 1.

\section{Procedure (bioavailability assessment)}

On each study morning, the participants visited the laboratory at 08.30 hours. Venous blood samples were collected using $4.7 \mathrm{ml}$ monovettes (containing lithium heparin) before the administration of the day's treatment and then 45, 90 and 120 min after the administration of treatment. The samples were centrifuged at $2500 \mathrm{rpm}$ for $15 \mathrm{~min}$ at $20^{\circ} \mathrm{C}$ to obtain plasma, which was then stored at $-80^{\circ} \mathrm{C}$ until analysis.

\section{Preparation of samples}

Samples were handled in low-light conditions to reduce the scope for isomerisation. Plasma samples were defrosted at room temperature immediately before extraction, vortexed and then sonicated for $5 \mathrm{~min}$. A $200 \mu \mathrm{l}$ aliquot was mixed with $900 \mu \mathrm{l}$ of HPLC-grade ethanol plus $0 \cdot 1 \%$ formic acid $(\mathrm{v} / \mathrm{v})$, along with $100 \mu \mathrm{l}$ of naringenin internal standard (IS1; Extrasynthese) in ethanol $(500 \mathrm{ng} / \mathrm{ml})$. The samples were vortexed, sonicated and then separated via micro-centrifugation at $17000 \mathrm{~g}$ for $10 \mathrm{~min}$. The supernatant was removed and placed in an amber $1.5 \mathrm{ml}$ centrifuge tube (Eppendorf). The pellet was re-extracted with $1.2 \mathrm{ml}$ of $83 \%$ aqueous ethanol $(\mathrm{v} / \mathrm{v})$ following the same protocol. Both extracts were evaporated to dryness under vacuum using a centrifugal evaporator (EZ2+; Genevac) and frozen at $-20^{\circ} \mathrm{C}$. On the day of analysis, a $70 \mu \mathrm{l}$ portion of ethanol was added to the secondary extract, which was vortexed and sonicated. A $50 \mu \mathrm{l}$ aliquot of this solution was then added to the primary extract, which following vortexing and sonication was mixed with $50 \mu \mathrm{l}$ taxifolin (IS2 at $2 \mu \mathrm{g} / \mathrm{ml}$; Extrasynthese) in $0.2 \%$ ascorbic acid solution. This solution was vortexed and separated by centrifugation, and the supernatant was placed in an amber vial and analysed via liquid chromatography-MS. Extractions were made in duplicate for each time point. To test the extraction efficiency of this method, blank plasma was spiked with standards at $50 \mathrm{~nm}, 500 \mathrm{~nm}, 5 \mu \mathrm{m}$ and $10 \mu \mathrm{m}$ concentrations. Across this range, the average extraction efficiencies for trans-resveratrol (Cayman Chemicals), resveratrol 3-O-sulphate, resveratrol 4'-O-glucuronide and resveratrol 3-O-glucuronide (Bertin Pharma) were 74, 72, 52 and 55\%, respectively. IS1 and IS2 were extracted consistently at 82 and $100 \%$, respectively.

\section{Liquid chromatography-MS analysis}

Liquid chromatography-MS analysis was conducted using a Shimadzu LC2010CHT HPLC system, consisting of an integrated quaternary pump, a degasser, a chilled autosampler $\left(8^{\circ} \mathrm{C}\right)$ and a column oven $\left(30^{\circ} \mathrm{C}\right)$, connected to an LCMS2020 single quadrupole mass spectrometer. A $10 \mu \mathrm{l}$ sample aliquot was separated on an XDB-C18 $1.8 \mu \mathrm{m}, 4.6 \times 50 \mathrm{~mm}$ column (Agilent), running a binary gradient of liquid chromatography-MS-grade water $v$. acetonitrile, both containing $0 \cdot 1 \%$ formic acid $(\mathrm{v} / \mathrm{v})$, running at $0.5 \mathrm{ml} / \mathrm{min}$. The gradient started at $5 \%$ acetonitrile and moved to $10 \%$ at $5 \mathrm{~min}, 40 \%$ at $20 \mathrm{~min}$ and $90 \%$ at $25 \mathrm{~min}$. Following $4 \mathrm{~min}$ of washing, the column returned to running $5 \%$ acetonitrile at $30 \mathrm{~min}$ and was re-equilibrated over $3 \mathrm{~min}$. The MS analysis was run with an interface temperature set to $350^{\circ} \mathrm{C}$, using nebuliser and drying gas flow rates of 1.5 and 15 litres $/ \mathrm{min}$, respectively. The analysis was carried out in a negative single-ion monitoring mode, following $\mathrm{m} / z$ of 403 (glucuronides), 307 (sulphates), 271 (naringenin IS1), 303 (taxifolin IS2) and 227 (aglycone resveratrol). A persistent formate adduct of aglycone resveratrol $(\mathrm{m} / z$ 273) was also followed as a qualifying ion. The limit of quantification was $16 \mathrm{~nm}$ for glucuronides, $22 \mathrm{~nm}$ for sulphates, and 145 and $290 \mathrm{~nm}$ for cis- and transaglycone resveratrol, respectively. Peak areas were normalised to that of IS2 for quantification, while IS1 was used to judge individual sample extraction. The retention times of cisisomer resveratrol conjugates were identified by subjecting commercially available trans-isomers $(10 \mu \mathrm{g} / \mathrm{ml}$ in $50 \%$ aqueous ethanol, plus $0.1 \%$ ascorbic acid and $0.05 \%$ formic acid) to ultraviolet light $(254 \mathrm{~nm})$ for $4 \mathrm{~h}$. Cis-isomer resveratrol conjugates were quantified as trans-isomer equivalents and then summed with the corresponding trans-isomers.

\section{Statistical analyses}

The analyses of plasma data were carried out with SPSS 16.0 for Windows (SPSS, Inc.) using within-subjects ANOVA (treatment $\times$ time) for each metabolite and paired-samples $t$ tests to compare AUC, $C_{\max }$ and $T_{\max }$, between the two treatments, for each metabolite.

NIRS data were analysed with Minitab 16 for Windows (Minitab, Inc.). For each variable (oxy-Hb, deoxy-Hb and total $\mathrm{Hb}$ ), data were converted to 'change from baseline' (calculated from a $10 \mathrm{~min}$ pre-treatment resting period) and averaged across $2 \mathrm{~min}$ epochs during the $40 \mathrm{~min}$ 'rest/absorption' and 40 min cognitive task performance periods. The analysis was based on an average of the two NIRS channels to give a measure of cerebral haemodynamics across the prefrontal cortex as a whole, in line with the method of Kennedy et al. ${ }^{(10)}$.

The primary analysis of the averaged NIRS data was conducted using within-subjects ANOVA (treatment $\times 2$ min epoch) with a priori planned comparisons of data from each epoch being made between placebo and each of the resveratrol treatment groups (250 mg resveratrol and $250 \mathrm{mg}$ resveratrol with $20 \mathrm{mg}$ piperine) using $t$ tests calculated with the mean squares error from the ANOVA ${ }^{(26)}$. To protect against the possibility of type 1 errors, planned comparisons are only reported if they evinced a consistent pattern of significant effects across the analysis period.

Task performance data (also analysed with SPSS 16.0) were analysed as change from pre-dose baseline for each individual task (serial $7 \mathrm{~s}$, RVIP, serial 13 s, 3-back and serial 17s) using within-subjects ANOVA (treatment $\times$ repetition), with planned comparisons for data from each repetition being made as described above.

A power calculation conducted using $G^{*}$ Power ${ }^{(27)}$ suggested that a sample size of twenty-four would be adequate to have greater than an $80 \%$ chance of detecting 
the medium effect sizes demonstrated in previous research assessing the effect of resveratrol on NIRS parameters ${ }^{(10)}$

\section{Results}

\section{Near-IR spectroscopy parameters}

Total $\mathrm{Hb}$. The ANOVA of total $\mathrm{Hb}$ data revealed that there was a significant interaction between the post-dose epoch and treatment $(P<0 \cdot 01)$. Planned comparisons revealed that, compared with placebo, treatment with $250 \mathrm{mg}$ resveratrol failed to elicit any modulation of total $\mathrm{Hb}$ levels. However, following treatment with $250 \mathrm{mg}$ resveratrol combined with $20 \mathrm{mg}$ piperine, although there were no significant effects during the absorption period, total $\mathrm{Hb}$ levels were significantly increased for all task performance epochs (apart from 45, 51 and $79 \mathrm{~min}$ ). Time points 41, 49 and 61 were all significant at the 0.05 level and the remainder at the $0 \cdot 01$ level.

Oxygenated $\mathrm{Hb}$ (oxy-Hb). The ANOVA of oxy-Hb data revealed that there was a significant interaction between the post-dose epoch and treatment $(P<0.05)$. The pattern was similar to that observed for total $\mathrm{Hb}$, with no modulation being observed following treatment with $250 \mathrm{mg}$ resveratrol, but with significantly increased oxy-Hb levels being observed following treatment with $250 \mathrm{mg}$ resveratrol combined with $20 \mathrm{mg}$ piperine (all epochs, $P<0 \cdot 01$; epochs 45, 49 and 51 , $P<0 \cdot 05$; and epoch 79, $P=$ NS).

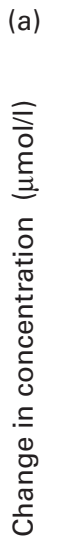

Absorption period

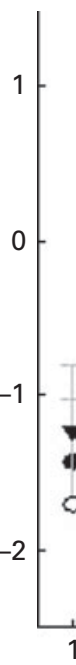

(b)

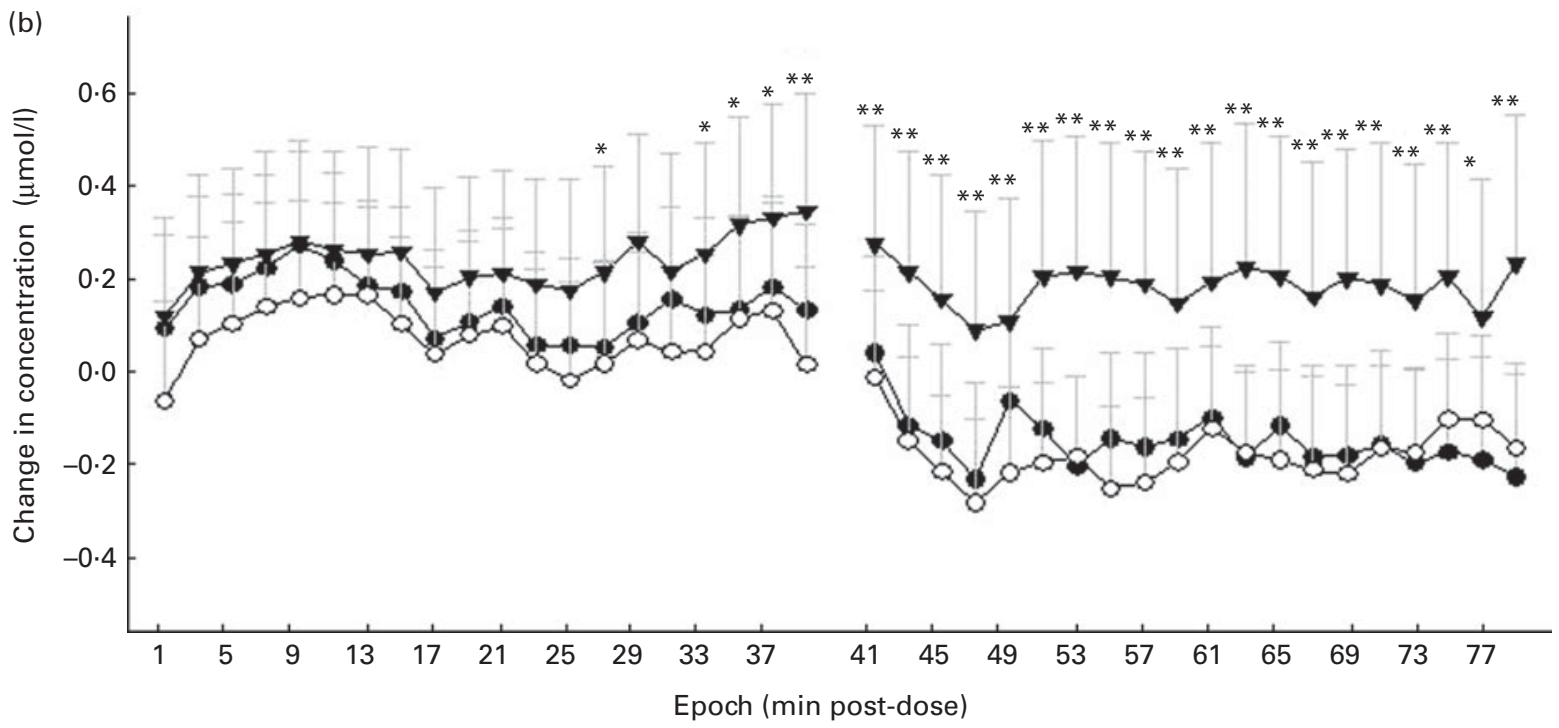

Fig. 2. Haemodynamic effects of $250 \mathrm{mg}$ of trans-resveratrol alone and when co-supplemented with 20 mg of piperine in healthy, young human subjects. Changes in the concentrations of (a) total $\mathrm{Hb}$ and (b) deoxygenated $\mathrm{Hb}$ (deoxy-Hb) during a 40 min absorption period and subsequent 40 min of cognitive task performance following the administration of placebo $(O), 250 \mathrm{mg}$ trans-resveratrol $(\bullet)$ and $250 \mathrm{mg}$ trans-resveratrol with $20 \mathrm{mg}$ piperine $(\mathbf{\nabla})$. The study followed a cross-over design ( $n 23$ per condition). Data were averaged across 2 min epochs. A priori planned comparisons between data from each resveratrol group and those from the placebo group for each epoch were made using $t$ tests by incorporating mean squares error from an initial ANOVA. Values are means, with their standard errors represented by vertical bars. Mean value was significantly different from that of the placebo group: ${ }^{*} P<0.05,{ }^{* \star} P<0.01$. 
Table 1. Effects of resveratrol on cognitive performance

(Mean values with their standard errors; $n$ 23)

\begin{tabular}{|c|c|c|c|c|c|c|c|c|c|c|c|c|c|c|}
\hline \multirow[b]{3}{*}{ Measures } & \multirow[b]{3}{*}{ Treatment condition } & \multicolumn{10}{|c|}{ Task battery repetition } & & & \\
\hline & & \multicolumn{2}{|c|}{ Baseline } & \multicolumn{2}{|c|}{1} & \multicolumn{2}{|l|}{2} & \multicolumn{2}{|c|}{3} & \multicolumn{2}{|l|}{4} & \multicolumn{3}{|c|}{ ANOVA } \\
\hline & & Mean & SEM & Mean & SEM & Mean & SEM & Mean & SEM & Mean & SEM & Effect & $F$ & $P$ \\
\hline \multirow{3}{*}{$7 \mathrm{~s}$ Correct $(n)$} & $250 \mathrm{mg}$ resveratrol & 28.85 & 2.75 & $1 \cdot 20$ & 1.02 & 1.98 & 0.94 & 1.54 & 0.83 & 0.80 & $1 \cdot 16$ & $\mathrm{~T}$ & 0.252 & 0.778 \\
\hline & $\begin{array}{l}250 \mathrm{mg} \text { resveratrol with } 20 \mathrm{mg} \\
\text { piperine }\end{array}$ & $28 \cdot 83$ & $2 \cdot 59$ & 1.52 & $0 \cdot 85$ & -0.04 & 0.94 & 0.39 & $1 \cdot 25$ & 0.57 & $1 \cdot 13$ & $\mathrm{R}$ & 0.487 & 0.692 \\
\hline & Placebo & 28.85 & $2 \cdot 04$ & 1.94 & $1 \cdot 12$ & 0.89 & $1 \cdot 11$ & 0.11 & 1.43 & 0.98 & 1.29 & $\mathrm{~T} \times \mathrm{R}$ & 0.675 & 0.606 \\
\hline \multirow[t]{3}{*}{$7 \mathrm{~s}$ Incorrect $(n)$} & $250 \mathrm{mg}$ resveratrol & 1.87 & 0.30 & 0.35 & 0.51 & -0.26 & 0.37 & 0.35 & 0.38 & 0.70 & 0.55 & $\mathrm{~T}$ & 0.517 & 0.600 \\
\hline & $\begin{array}{l}250 \mathrm{mg} \text { resveratrol with } 20 \mathrm{mg} \\
\text { piperine }\end{array}$ & $1 \cdot 67$ & 0.23 & 0.11 & $0 \cdot 39$ & 0.67 & 0.38 & 1.33 & 0.52 & 1.07 & 0.49 & $\mathrm{R}$ & $2 \cdot 09$ & $0 \cdot 110$ \\
\hline & Placebo & 1.91 & 0.26 & 0.30 & 0.52 & 0.30 & 0.47 & 0.78 & 0.60 & 0.13 & 0.46 & $\mathrm{~T} \times \mathrm{R}$ & 1.02 & 0.416 \\
\hline \multirow[t]{3}{*}{ 13s Correct $(n)$} & $250 \mathrm{mg}$ resveratrol & $22 \cdot 22$ & $2 \cdot 25$ & $0 \cdot 70$ & 0.88 & -0.78 & 0.90 & 0.22 & 0.87 & $-1 \cdot 17$ & 0.98 & $\mathrm{~T}$ & 1.68 & 0.199 \\
\hline & $\begin{array}{l}250 \mathrm{mg} \text { resveratrol with } 20 \mathrm{mg} \\
\text { piperine }\end{array}$ & 22.46 & $2 \cdot 17$ & $1 \cdot 33$ & 0.75 & $-1 \cdot 15$ & 1.26 & -0.11 & $1 \cdot 25$ & 1.07 & 0.87 & $\mathrm{R}$ & $3 \cdot 17$ & $0.030^{*}$ \\
\hline & Placebo & $21 \cdot 83$ & 1.60 & 3.26 & 0.83 & 0.78 & 1.41 & $1 \cdot 17$ & $1 \cdot 20$ & 1.09 & 1.03 & $\mathrm{~T} \times \mathrm{R}$ & 0.644 & 0.695 \\
\hline \multirow{3}{*}{13 s Incorrect $(n)$} & $250 \mathrm{mg}$ resveratrol & $2 \cdot 04$ & 0.23 & 0.13 & 0.40 & $2 \cdot 04$ & 1.00 & 0.65 & 0.51 & $1 \cdot 17$ & 0.47 & $\mathrm{~T}$ & 0.969 & 0.388 \\
\hline & $\begin{array}{l}250 \mathrm{mg} \text { resveratrol with } 20 \mathrm{mg} \\
\text { piperine }\end{array}$ & 1.89 & 0.36 & $0 \cdot 11$ & 0.44 & 1.59 & 0.94 & 1.59 & 0.63 & $0 \cdot 76$ & 0.73 & $\mathrm{R}$ & $7 \cdot 08$ & $<0.001^{\star *}$ \\
\hline & Placebo & $2 \cdot 39$ & 0.36 & -1.09 & 0.33 & 0.96 & 0.84 & 0.78 & 0.53 & 0.44 & 0.58 & $\mathrm{~T} \times \mathrm{R}$ & 0.445 & 0.765 \\
\hline \multirow[t]{3}{*}{17 s Correct $(n)$} & $250 \mathrm{mg}$ resveratrol & $17 \cdot 22$ & 1.68 & 1.39 & 0.71 & 1.48 & 0.81 & 2.35 & 0.75 & 1.09 & 1.13 & $\mathrm{~T}$ & 0.405 & 0.670 \\
\hline & $\begin{array}{l}250 \mathrm{mg} \text { resveratrol with } 20 \mathrm{mg} \\
\text { piperine }\end{array}$ & $17 \cdot 78$ & $1 \cdot 61$ & 0.39 & 0.63 & 0.44 & 0.86 & 0.87 & 0.90 & $2 \cdot 13$ & 0.76 & $\mathrm{R}$ & 0.502 & 0.638 \\
\hline & Placebo & $16 \cdot 80$ & 1.29 & $1 \cdot 72$ & 0.62 & 1.37 & 0.68 & 1.15 & 0.95 & 1.89 & 0.59 & $\mathrm{~T} \times \mathrm{R}$ & 1.07 & 0.383 \\
\hline \multirow{3}{*}{$17 \mathrm{~s}$ Incorrect $(n)$} & $250 \mathrm{mg}$ resveratrol & $2 \cdot 28$ & 0.28 & 0.15 & 0.41 & 0.02 & 0.47 & 0.24 & 0.52 & 1.54 & 1.09 & $\mathrm{~T}$ & 0.719 & 0.493 \\
\hline & $\begin{array}{l}250 \mathrm{mg} \text { resveratrol with } 20 \mathrm{mg} \\
\text { piperine }\end{array}$ & $2 \cdot 17$ & 0.29 & 0.30 & 0.42 & $0 \cdot 30$ & 0.50 & 0.57 & 0.42 & 0.52 & 0.37 & $\mathrm{R}$ & 1.41 & 0.254 \\
\hline & Placebo & 2.57 & 0.27 & -0.30 & 0.37 & -0.44 & 0.45 & 0.44 & 0.67 & -0.04 & 0.36 & $\mathrm{~T} \times \mathrm{R}$ & 0.791 & 0.578 \\
\hline \multirow[t]{3}{*}{$N$-back accuracy (\%) } & $250 \mathrm{mg}$ resveratrol & 93.38 & $1 \cdot 17$ & -0.34 & 0.97 & -1.02 & 1.05 & -0.92 & 1.08 & -0.05 & 1.00 & $\mathrm{~T}$ & 0.617 & 0.544 \\
\hline & $\begin{array}{l}250 \mathrm{mg} \text { resveratrol with } 20 \mathrm{mg} \\
\text { piperine }\end{array}$ & 94.40 & 0.91 & -2.03 & 1.02 & $-1 \cdot 84$ & 1.09 & -0.29 & 0.89 & -1.45 & $1 \cdot 27$ & $\mathrm{R}$ & 0.274 & 0.844 \\
\hline & Placebo & 94.40 & 0.74 & -1.26 & 1.08 & -1.55 & 0.92 & $-2 \cdot 61$ & $1 \cdot 13$ & 01.45 & 0.93 & $\mathrm{~T} \times \mathrm{R}$ & 0.678 & 0.599 \\
\hline \multirow{3}{*}{$\begin{array}{l}N \text {-back reaction time } \\
\quad(\mathrm{ms})\end{array}$} & $250 \mathrm{mg}$ resveratrol & $1540 \cdot 45$ & $145 \cdot 80$ & -291.04 & $48 \cdot 75$ & $-345 \cdot 87$ & 53.98 & -312.95 & 52.58 & $-398 \cdot 24$ & 58.12 & $\mathrm{~T}$ & 1.28 & 0.288 \\
\hline & $\begin{array}{l}250 \mathrm{mg} \text { resveratrol with } 20 \mathrm{mg} \\
\text { piperine }\end{array}$ & $1476 \cdot 26$ & 189.03 & -243.72 & 67.01 & $-287 \cdot 30$ & $77 \cdot 69$ & -375.74 & 94.44 & $-292 \cdot 16$ & 70.96 & $\mathrm{R}$ & 3.93 & $0.012^{*}$ \\
\hline & Placebo & 1475.04 & 161.35 & $-194 \cdot 12$ & 34.69 & $-149 \cdot 39$ & $70 \cdot 65$ & -264.79 & 81.89 & -271.44 & 57.14 & $T \times R$ & $1 \cdot 12$ & 0.347 \\
\hline \multirow{3}{*}{ RVIP correct (\%) } & $250 \mathrm{mg}$ resveratrol & 71.06 & 3.76 & 0.41 & $2 \cdot 98$ & -4.48 & 2.44 & -7.47 & 3.73 & -7.76 & $2 \cdot 58$ & $\mathrm{~T}$ & $1 \cdot 17$ & 0.321 \\
\hline & $\begin{array}{l}250 \mathrm{mg} \text { resveratrol with } 20 \mathrm{mg} \\
\text { piperine }\end{array}$ & $65 \cdot 81$ & 4.00 & $3 \cdot 76$ & $2 \cdot 32$ & $1 \cdot 31$ & $3 \cdot 39$ & $-4 \cdot 36$ & $3 \cdot 39$ & $-1 \cdot 68$ & 3.51 & $\mathrm{R}$ & $7 \cdot 58$ & $<0.001^{* *}$ \\
\hline & Placebo & $69 \cdot 16$ & 3.90 & 1.50 & $2 \cdot 25$ & $-7 \cdot 38$ & 3.65 & -7.47 & $2 \cdot 51$ & -6.66 & 3.40 & $\mathrm{~T} \times \mathrm{R}$ & 0.489 & 0.816 \\
\hline
\end{tabular}

$\mathrm{T}$, treatment; $\mathrm{R}$, repetition; RVIP, rapid visual information processing

There was a significant main effect for $\mathrm{R}:{ }^{*} P<0 \cdot 05,{ }^{* *} P<0.01$. 
Deoxygenated $\mathrm{Hb}$ (deoxy-Hb). The ANOVA of deoxy-Hb data revealed that there was no significant main effect or interaction between time and treatment. Planned comparisons, however, demonstrated a consistent pattern of significant effects, which began to emerge during the end of the absorption phase and continued throughout the post-dose task period. After treatment with $250 \mathrm{mg}$ resveratrol combined with $20 \mathrm{mg}$ piperine, deoxy-Hb levels were significantly increased in comparison with those observed after placebo administration (absorption period: epochs 27, 29, 33, 35 and $37, P<0.05$ and epoch 39, $P<0.01$; post-dose task period: all epochs, $P<0.01$ and epoch 77, $P<0.05)$.

The mean data (with their standard errors) and the results of the planned comparisons for total $\mathrm{Hb}$ and deoxy-Hb are shown in Fig. 2.

\section{Cognitive task performance and mood}

There were no significant treatment-related differences in any cognitive or mood measures. The raw baseline task scores and mood ratings and changes from baseline mean task scores and mood ratings are given in Tables 1 and 2, respectively.

\section{Blood pressure}

No significant treatment-related differences were observed in pulse rate and diastolic or systolic blood pressure. The raw baseline blood pressure readings and changes from baseline post-dose blood pressure readings are given in Table 3 .

\section{Bioavailability}

No resveratrol (in any form) was found in baseline samples, indicating that none of the participants consumed resveratrol before the start of the study. Following oral intervention with $250 \mathrm{mg}$ of resveratrol, plasma concentrations of total resveratrol metabolites ranged from 2 to $18.2 \mu \mathrm{M}$, varying between the participants and treatments. However, no aglycone trans- or cis-resveratrol was quantifiable in plasma. Resveratrol 3-O-sulphate was the predominant metabolite in all participants, contributing $59-81 \%$ of total metabolites. The 4'-O-glucuronide and 3-O-glucuronide forms made roughly equal contributions to the remaining metabolites in circulation. $C_{\max }$ was typically achieved at $90 \mathrm{~min}$. Resveratrol conjugates were present in plasma as both trans-isomers and $c i s$-isomers, varying between the participants. The average $C_{\max }$ trans:cis ratios for resveratrol 3-O-sulphate and resveratrol 3-O-glucuronide following the consumption of all trans-resveratrol were 4.7 (SEM 5.6) (range 1.2-15.9) and 5.1 (SEM 5.6) (range 0.94-18.8), respectively. Cis-resveratrol $4^{\prime}$-O-glucuronide was found in some, but not in all subjects. Extraction efficiency tests did not indicate significant induction of isomerisation during sample handling, suggesting that this conversion occurs in vivo.

Although the average concentrations of resveratrol 3-O-sulphate, $4^{\prime}$-O-glucuronide and 3-O-glucuronide at $C_{\max }$ appeared to be lower following the co-supplementation of resveratrol with piperine compared with those following supplementation of resveratrol alone, there was no significant difference between the treatments. Similarly, there was no significant

Table 2. Effects of $250 \mathrm{mg}$ resveratrol alone and when co-supplemented with $20 \mathrm{mg}$ piperine on mood in healthy, young human subjects (Mean values with their standard errors; $n$ 23)

\begin{tabular}{|c|c|c|c|c|c|c|c|c|}
\hline \multirow[b]{2}{*}{ Measures } & \multirow[b]{2}{*}{ Treatment condition } & \multicolumn{2}{|c|}{ Baseline } & \multicolumn{2}{|c|}{ Post-dose } & \multicolumn{3}{|c|}{ ANOVA } \\
\hline & & Mean & SEM & Mean & SEM & Effect & $F$ & $P$ \\
\hline \multirow[t]{3}{*}{ Alert } & $250 \mathrm{mg}$ resveratrol & $50 \cdot 83$ & 3.79 & -6.65 & 5.44 & $\mathrm{~T}$ & 0.767 & 0.470 \\
\hline & $250 \mathrm{mg}$ resveratrol with $20 \mathrm{mg}$ piperine & $49 \cdot 13$ & 3.78 & 4.43 & 4.07 & $\mathrm{R}$ & 0.359 & 0.555 \\
\hline & Placebo & 51.57 & 4.08 & -4.87 & 4.68 & $T \times R$ & $3 \cdot 28$ & $0.047^{*}$ \\
\hline \multirow[t]{3}{*}{ Jittery } & $250 \mathrm{mg}$ resveratrol & $16 \cdot 83$ & 2.91 & $19 \cdot 78$ & 5.40 & $\mathrm{~T}$ & 0.532 & 0.591 \\
\hline & $250 \mathrm{mg}$ resveratrol with $20 \mathrm{mg}$ piperine & $18 \cdot 61$ & 3.33 & $20 \cdot 48$ & 4.95 & $\mathrm{R}$ & $25 \cdot 79$ & $<0.001^{\star \star}$ \\
\hline & Placebo & $15 \cdot 39$ & 2.54 & $20 \cdot 87$ & 4.73 & $T \times R$ & 0.022 & 0.979 \\
\hline \multirow[t]{3}{*}{ Mental fatigue } & $250 \mathrm{mg}$ resveratrol & 28.96 & 4.69 & 35.65 & $6 \cdot 18$ & $\mathrm{~T}$ & 0.839 & 0.439 \\
\hline & $250 \mathrm{mg}$ resveratrol with $20 \mathrm{mg}$ piperine & 27.48 & 4.86 & 32.48 & 5.93 & $\mathrm{R}$ & $45 \cdot 47$ & $<0.001^{\star \star}$ \\
\hline & Placebo & $26 \cdot 22$ & $4 \cdot 10$ & 33.74 & $6 \cdot 11$ & $T \times R$ & 0.147 & 0.864 \\
\hline \multirow[t]{3}{*}{ Overall mood } & $250 \mathrm{mg}$ resveratrol & $62 \cdot 87$ & 3.46 & $-16 \cdot 13$ & 4.48 & $\mathrm{~T}$ & $2 \cdot 66$ & $0.081 \mathrm{t}$ \\
\hline & $250 \mathrm{mg}$ resveratrol with $20 \mathrm{mg}$ piperine & 64.48 & 3.04 & $-12 \cdot 78$ & 3.60 & $\mathrm{R}$ & $25 \cdot 87$ & $<0.001^{\star \star}$ \\
\hline & Placebo & 67.35 & $2 \cdot 71$ & $-13 \cdot 74$ & 2.97 & $T \times R$ & 0.321 & 0.727 \\
\hline \multirow[t]{3}{*}{ Relaxed } & $250 \mathrm{mg}$ resveratrol & 62.91 & 2.67 & -24.52 & $5 \cdot 62$ & $\mathrm{~T}$ & 0.566 & 0.572 \\
\hline & $250 \mathrm{mg}$ resveratrol with $20 \mathrm{mg}$ piperine & 60.35 & 3.29 & $-14 \cdot 13$ & 6.00 & $\mathrm{R}$ & $20 \cdot 70$ & $<0.001^{\star \star}$ \\
\hline & Placebo & 62.52 & 1.98 & $-20 \cdot 61$ & 4.44 & $T \times R$ & 1.79 & 0.179 \\
\hline \multirow[t]{3}{*}{ Tense } & $250 \mathrm{mg}$ resveratrol & $25 \cdot 48$ & 3.29 & $25 \cdot 74$ & 6.35 & $\mathrm{~T}$ & $2 \cdot 32$ & 0.110 \\
\hline & $250 \mathrm{mg}$ resveratrol with $20 \mathrm{mg}$ piperine & 23.87 & 3.28 & $26 \cdot 35$ & $6 \cdot 40$ & $\mathrm{R}$ & 26.08 & $<0.001^{\star \star}$ \\
\hline & Placebo & 19.83 & 3.02 & $25 \cdot 30$ & 5.37 & $T \times R$ & 0.016 & 0.984 \\
\hline \multirow[t]{3}{*}{ Tired } & $250 \mathrm{mg}$ resveratrol & 47.09 & 4.51 & 14.57 & 5.33 & $\mathrm{~T}$ & 0.405 & 0.669 \\
\hline & $250 \mathrm{mg}$ resveratrol with $20 \mathrm{mg}$ piperine & $50 \cdot 74$ & 5.05 & 4.04 & 3.92 & $\mathrm{R}$ & 5.96 & $0.023^{*}$ \\
\hline & Placebo & $45 \cdot 57$ & 4.42 & 11.52 & $6 \cdot 39$ & $T \times R$ & 1.72 & 0.191 \\
\hline
\end{tabular}

$\mathrm{T}$, treatment; $\mathrm{R}$, repetition; $\mathrm{t}$, trend.

There were significant main effects for $\mathrm{R}$ and the $\mathrm{T} \times \mathrm{R}$ interaction: ${ }^{\star} P<0.05,{ }^{\star \star} P<0.01$. 
difference in the values of area under the curve, and there was no significant change in $T_{\max }$ between the treatments.

The mean plasma concentrations of trans-resveratrol 3-Osulphate and combined 4'-O-glucuronide and 3-O-glucuronide metabolites at pre-treatment and at 45,90 and $120 \mathrm{~min}$ postdose time points, for both treatments, are shown in Fig. 3.

\section{Discussion}

The present study demonstrates that the well-established bioenhancer piperine can increase the bioefficacy of the polyphenol resveratrol when co-supplemented in healthy human subjects. Whereas $250 \mathrm{mg}$ of orally administered trans-resveratrol had no significant effects on overall CBF (total $\mathrm{Hb}$ ) during the performance of cognitively demanding tasks, co-administration of the same dose of resveratrol with $20 \mathrm{mg}$ piperine resulted in significantly increased CBF for the duration of the $40 \mathrm{~min}$ post-dose task period. The findings with regard to the supplementation of resveratrol alone in this respect are broadly in line with the dose-response pattern of $\mathrm{CBF}$ observed following resveratrol administration in a previous study, in which a dose of $250 \mathrm{mg}$ was largely ineffective ${ }^{(10)}$. Despite this piperine-mediated enhancement of the CBF effects of resveratrol, there were no significant treatment-related differences in the performance of the cognitive tasks, blood pressure/heart rate or participants' ratings of mood for either active treatment.

The pattern of haemodynamic effects of resveratrol observed in the present study, when supplemented with piperine, is exactly in line with that in the aforementioned previous resveratrol intervention study following the administration of a $500 \mathrm{mg}$ dose ${ }^{(10)}$. This pattern is observed as significantly higher levels of total $\mathrm{Hb}$ and oxy- $\mathrm{Hb}$, alongside deoxy-Hb, during the post-dose cognitive task period and represents increased $\mathrm{CBF}$ and oxygen utilisation, respectively. This haemodynamic response is dissimilar to that observed during cognitive task performance alone. Here, total $\mathrm{Hb}$ and oxy-Hb levels typically rise alongside a concomitant decline in deoxy-Hb levels ${ }^{(28)}$, with this phenomenon being predicated based on the fact that neural activation instigates an increase in $\mathrm{CBF}$ that is greater than the metabolic rate of oxygen extraction/utilisation. As such, deoxy-Hb levels can be observed to decrease during cognitive performance ${ }^{(29)}$. The different deoxy-Hb response observed following resveratrol treatment is probably predicated based on indirect effects on mitochondrial phosphorylation. In support of this, Lagouge et $a l{ }^{(30)}$ reported that in mice supplemented with $400 \mathrm{mg} / \mathrm{kg} / \mathrm{d}$ resveratrol, for 15 weeks, significantly increased mitochondrial structures and enzymatic activity. This resulted in a significant increase in $\mathrm{VO}_{2}$ and $\mathrm{VO}_{2 \max }$ rates and was observed to increase running time and tolerance to cold. In terms of mechanisms, resveratrol can interact with the sirtuin ('silent information regulator'; SIRT) system, a class of proteins involved in multifarious biological processes that has received a great amount of attention over the past decade in relation to life extension ${ }^{(31)}$. Of importance here is that SIRT is implicated in the deacetylation of PPAR $\gamma$ co-activator $1-\alpha(P G C-1 \alpha)$, a gene that controls mitochondrial biogenesis and function ${ }^{(32)}$, 
(a)

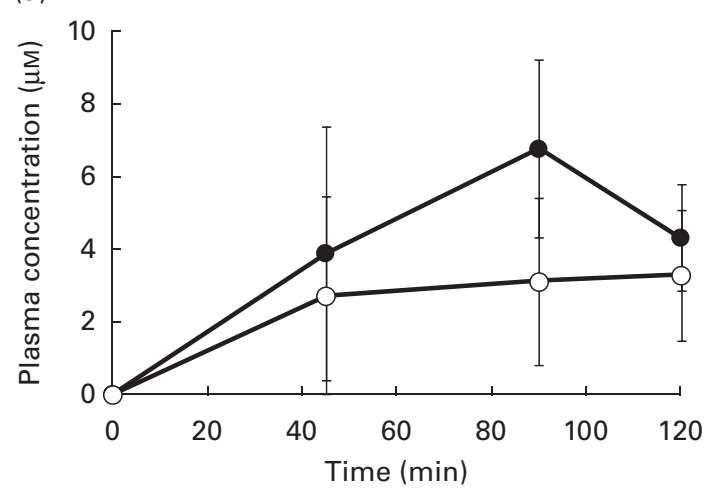

(b)

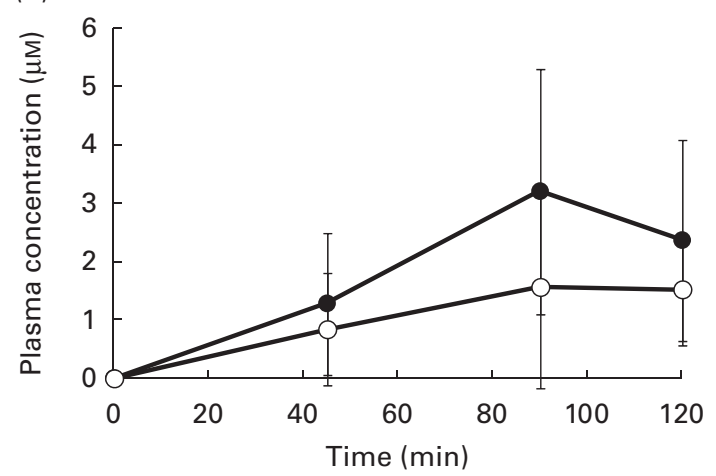

Fig. 3. Plasma bioavailability of resveratrol metabolites following (a) the administration of $250 \mathrm{mg}$ trans-resveratrol alone and (b) the administration of $250 \mathrm{mg}$ trans-resveratrol with $20 \mathrm{mg}$ piperine in healthy, young human subjects. Values are means $(n 6)$, with their standard errors represented by vertical bars.

-, Concentration of resveratrol 3-O-sulphate; $O$, combined concentrations of resveratrol 4'-O-glucuronide and resveratrol 3-O-glucuronide.

and while the oxygenation effects in the above-mentioned study in rodents were observed following chronic consumption, these mechanisms would explain the $\mathrm{VO}_{2}$ effects observed in the present study, represented by deoxy-Hb.

Interestingly, in light of the significant CBF effects occurring only with the resveratrol/piperine combination, no significant differences were observed in the plasma concentrations of resveratrol between the treatments. In both treatment conditions, resveratrol metabolites were present in the plasma across the post-dose cognitive task period and the parent compound was unquantifiable at all time points. However, contrary to the hypothesis of piperine-induced bioenhancement, the pattern of effects observed in the present study actually suggests inhibition rather than enhancement of plasma concentrations; for example, the $C_{\max }$ of total metabolites after treatment with $250 \mathrm{mg}$ resveratrol was $9.98 \mu \mathrm{M}$ compared with $4.82 \mu \mathrm{M}$ in the piperine co-supplemented condition. Piperine also appeared to be inhibiting the transit of resveratrol, evidenced by the $T_{\max }$ of metabolites in the $250 \mathrm{mg}$ resveratrol condition occurring at the 90 min sample time point compared with the $120 \mathrm{~min}$ time point in the co-supplemented condition and the observation of metabolite concentrations reducing at the $120 \mathrm{~min}$ time point in the $250 \mathrm{mg}$ resveratrol condition and not in the co-supplemented condition. Nevertheless, this pattern of effects exhibited no significant differences between the treatment groups, which suggests two possibilities: either piperine can exert CBF effects independently of resveratrol or, alternatively, it potentiates the effects of resveratrol observed previously on CBF.

Taking the first of these possibilities into account, it is notable that there is only one study ${ }^{(33)}$ that suggests that piperine is capable of interacting with $\mathrm{NO}$ and that this is the inducible NO synthase isoform that is stimulated in response to immunological stimuli ${ }^{(34)}$ and is not associated with cerebral vasorelaxation and increased blood flow. No data exist to suggest that piperine is capable of affecting oxygenation or indeed any other factor relevant to the present study, and this precluded the need for a piperine-only treatment condition in the study. The exception here is a small amount of literature in rats that suggests that chronic (up to 4 weeks) piperine supplementation might improve aspects of performance, although this appears to be mostly related to mood augmentation rather than to enhanced cognition per $s e^{(35-37)}$. Nevertheless, future studies investigating the efficacy of piperine alone on these parameters, in human subjects, to clarify this issue are warranted.

In light of a lack of evidence to suggest that piperine has any influence on parameters relevant to $\mathrm{CBF}$, and in the face of no significant modulation of CBF being observed in the resveratrol condition alone (a finding mirrored in the study of Kennedy et $a l^{(10)}$ with the same dose), it seems more likely that piperine increases the bioefficacy of resveratrol by potentiating its vasorelaxatory properties. In support of this, resveratrol is a well-validated vasorelaxatory mediator ${ }^{(7)}$ and, at a higher dose $(500 \mathrm{mg})$, can increase CBF in healthy human subjects ${ }^{(10)}$.

Of the potential mechanisms to explain the efficacyenhancing effects of piperine, one is that piperine is able to enhance the activity of resveratrol, the neuronal vasculature, and/or some other factor relevant to CBF through its thermogenic properties. As evidence of the heat-proffering properties of piperine, specifically in neural tissue, Reanmongkol et al. ${ }^{(38)}$ reported on the ability of piperine to stimulate the activity of ATPase (but inhibition of oxidative phoshorylation), which produces heat as a by-product ${ }^{(39)}$. Thermogenic increases in tissue activity have previously been proposed as an explanation for piperine-mediated increases in plasma $\beta$-carotene concentrations in human subjects ${ }^{(13)}$ via an increase in the absorption rate of the intestinal epithelium and, as a mechanism, could exist without piperine inducing an overall increase in the bioavailability of resveratrol: a phenomenon observed previously ${ }^{(11-14)}$, but not replicated in the present study.

In terms of behavioural effects, the results of the present study are in line with previous findings, i.e. a lack of any effect of a $250 \mathrm{mg}$ dose of resveratrol with regard to cognitive task performance ${ }^{(10)}$. One of the primary reasons for using piperine in the present study was to ascertain whether this well-established bioenhancer of polyphenols also induces the enhancement of resveratrol's bioefficacy, especially in terms of cognitive function due to the null effects reported previously. However, while the increase in CBF during task performance was potentiated by piperine, the pattern was largely the same as that observed following the administration of 
a larger dose of resveratrol $\left(500 \mathrm{mg}\right.$ in Kennedy et $a{ }^{(10)}$ ), where cognitive effects were also lacking. Therefore, it would appear that acute increases in CBF are not sufficient, in themselves, to alter cognitive function in the young, healthy cohorts examined in the present study and previously. However, it may be the case that longer-term supplementation is required or indeed that the effects might translate into cognitive benefits in populations exhibiting age- or pathology-related decrements in $\mathrm{CBF}$ and cognitive function.

In conclusion, this is the first study to report that co-supplementation of piperine with resveratrol enhances the bioefficacy of resveratrol with regard to CBF effects in healthy human subjects, but not cognitive performance, and does this without altering the overall bioavailability of resveratrol in vivo.

\section{Supplementary material}

To view supplementary material for this article, please visit http://dx.doi.org/10.1017/S0007114514000737

\section{Acknowledgements}

The present study did not receive any financial support. The treatment substances and other materials were purchased on the open market.

The authors' contributions are as follows: E. L. W. collected the data and G. W. and T. P. D. planned and carried out the analysis of the plasma samples. All the authors were actively involved in the planning of the research and in the writing of the article and contributed to and reviewed the final publication.

None of the authors has any conflicts of interest to declare.

\section{References}

1. Fremont L (2000) Biological effects of resveratrol. Life Sci 66, 663-673.

2. Joseph JA, Fisher DR, Cheng V, et al. (2008) Cellular and behavioral effects of stilbene resveratrol analogues: implications for reducing the deleterious effects of aging. J Agric Food Chem 56, $10544-10551$.

3. Ritz MF, Ratajczak P, Curin Y, et al. (2008) Chronic treatment with red wine polyphenol compounds mediates neuroprotection in a rat model of ischemic cerebral stroke. $J$ Nutr 138, 519-525.

4. Gresele P, Pignatelli P, Guglielmini G, et al. (2008) Resveratrol, at concentrations attainable with moderate wine consumption, stimulates human platelet nitric oxide production. J Nutr 138, 1602-1608.

5. Rivera L, Morón R, Zarzuelo A, et al. (2009) Long-term resveratrol administration reduces metabolic disturbances and lowers blood pressure in obese Zucker rats. Biochem Pharmacol 77, 1053-1063.

6. Rush JWE, Quadrilatero J, Levy AS, et al. (2007) Chronic resveratrol enhances endothelium-dependent relaxation but does not alter eNOS levels in aorta of spontaneously hypertensive rats. Ex Biol Med (Maywood) 232, 814-822.

7. Wong R, Howe P, Buckley J, et al. (2011) Acute resveratrol supplementation improves flow-mediated dilatation in overweight/obese individuals with mildly elevated blood pressure. Nutr Metab Cardiovasc Dis 21, 851-856.
8. Moss MC, Scholey AB \& Wesnes K (1998) Oxygen administration selectively enhances cognitive performance in healthy young adults: a placebo-controlled double-blind crossover study. Psychopharmacology (Berl) 138, 27-33.

9. Scholey A, Harper S \& Kennedy D (2001) Cognitive demand and blood glucose. Physiol Behav 73, 585-592.

10. Kennedy DO, Wightman EL, Reay JL, et al. (2010) Effects of resveratrol on cerebral blood flow variables and cognitive performance in humans: a double-blind, placebo-controlled, crossover investigation. Am J Clin Nutr 91, 1590-1597.

11. Lambert JD, Hong J, Kim DH, et al. (2004) Piperine enhances the bioavailability of the tea polyphenol (-)-epigallocatechin-3-gallate in mice. J Nutr 134, 1948-1952.

12. Shoba G, Joy D, Joseph T, et al. (1998) Influence of piperine on the pharmacokinetics of curcumin in animals and human volunteers. Planta Medica 64, 353-356.

13. Badmaev V, Majeed M \& Norkus EP (1999) Piperine, an alkaloid derived from black pepper, increases serum response of beta-carotene during 14-days of oral betacarotene supplementation. Nutr Res 19, 381-388.

14. Johnson JJ, Nihal M, Siddiqui IA, et al. (2011) Enhancing the bioavailability of resveratrol by combining it with piperine. Mol Nutr Food Res 55, 1169-1176.

15. Reen RK, Jamwal DS, Taneja SC, et al. (1993) Impairment of UDP-glucose dehydrogenase and glucuronidation activities in liver and small intestine of rat and guinea pig in vitro by piperine. Biochem Pharmacol 46, 229-238.

16. Singh J, Dubey RK \& Atal CK (1986) Piperine-mediated inhibition of glucuronidation activity in isolated epithelial cells of the guinea-pig small intestine: evidence that piperine lowers the endogenous UDP-glucuronic acid content. J Pharmacol Exp Ther 236, 488-493.

17. van de Wetering K, Burkon A, Feddema W, et al. (2008) Intestinal BCRP/Bcrp1 and MRP3/Mrp3 are involved in the pharmacokinetics of resveratrol. Mol Pharmacol $\mathbf{7 5}$, 876-885.

18. Pollitt E, Leibel RL \& Greenfield D (1981) Brief fasting, stress, and cognition in children. Am J Clin Nutr 34, 1526-1533.

19. Pollitt E, Lewis NL, Garza C, et al. (1983) Fasting and cognitive function. J Psychiatr Res 17, 169-174.

20. Tian H-H, Aziz A-R, Png W, et al. (2011) Effects of fasting during Ramadan month on cognitive function in Muslim athletes. Asian J Sports Med 2, 145-153.

21. Green MW, Elliman NA \& Rogers PJ (1995) Lack of effect of short-term fasting on cognitive function. J Psychiatr Res 29, $245-253$.

22. Obrig H \& Villringer A (2003) Beyond the visible - imaging the human brain with light. J Cereb Blood Flow Metab 23, 1-18.

23. Kazui H, Kitagaki H \& Mori E (2000) Cortical activation during retrieval of arithmetical facts and actual calculation: a functional magnetic resonance imaging study. Psychiatry Clin Neurosci 54, 479-485.

24. Coull J, Frith C, Frackowiak RSJ, et al. (1996) A frontoparietal network for rapid visual information processing: a PET study of sustained attention and working memory. Neuropsychologia 34, 1085-1095.

25. Jansma JM, Ramsey NF, Coppola R, et al. (2000) Specific versus nonspecific brain activity in a parametric $N$-back task. Neuroimage 12, 688-697.

26. Keppel G (1991) Design and Analysis. New Jersey: Prentice Hall.

27. Faul F, Erdfelder E, Lang A-G, et al. (2007) G* Power 3: a flexible statistical power analysis program for the social, behavioral, and biomedical sciences. Behav Res Methods 39, 175-191. 
28. Steinbrink J, Villringer A, Kempf F, et al. (2005) Illuminating the BOLD signal: combined fMRI-fNIRS studies. Magn Reson Imaging 24, 495-505.

29. Hasegawa M, Carpenter PA \& Just MA (2002) An fMRI study of bilingual sentence comprehension and workload. Neuroimage 15, 647-660.

30. Lagouge M, Argmann C, Gerhart-Hines Z, et al. (2006) Resveratrol improves mitochondrial function and protects against metabolic disease by activating SIRT1 and PGC- $1 \alpha$. Cell 127, 1109-1122.

31. Baur JA, Pearson KJ, Price NL, et al. (2006) Resveratrol improves health and survival of mice on a high-calorie diet. Nature 444, 337-342.

32. Rodgers JT, Lerin C, Haas W, et al. (2005) Nutrient control of glucose homeostasis through a complex of PGC-1 and SIRT1. Nature 434, 113-118.

33. Vaibhav K, Shrivastava P, Javed H, et al. (2012) Piperine suppresses cerebral ischemia-reperfusion-induced inflammation through the repression of COX-2, NOS-2, and NF- $\mathrm{BB}$ in middle cerebral artery occlusion rat model. Mol Cell Biochem 367, 73-84.
34. Nathan C (1997) Inducible nitric oxide synthase: what difference does it make? J Clin Invest 100, 2417-2423.

35. Chonpathompikunlert P, Wattanathorn J \& Muchimapura S (2010) Piperine, the main alkaloid of Thai black pepper, protects against neurodegeneration and cognitive impairment in animal model of cognitive deficit like condition of Alzheimer's disease. Food Chem Toxicol 48, 798-802.

36. Priprem A, Chonpathompikunlert P, Sutthiparinyanont S, et al. (2011) Antidepressant and cognitive activities of intranasal piperine-encapsulated liposomes. Adv Biosci Biotechnol 2, 108-116.

37. Wattanathorn J, Chonpathompikunlert P, Muchimapura S, et al. (2008) Piperine, the potential functional food for mood and cognitive disorders. Food Chem Toxicol 46, 3106-3110.

38. Reanmongkol W, Janthasoot W, Wattanatorn W, et al. (1988) Effects of piperine on bioenergetic functions of isolated rat liver mitochondria. Biochem Pharmacol 37, 753-757.

39. Clapham J \& Arch J (2006) Thermogenic and metabolic antiobesity drugs: rationale and opportunities. Diabetes Obes Metab 9, 259-275. 